See discussions, stats, and author profiles for this publication at: https://www.researchgate.net/publication/333436267

\title{
An alternative update of the two-step QCA procedure
}

Article in Quality and Quantity · May 2019

DOI: 10.1007/s11135-019-00893-7

CITATIONS

0

1 author:

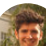

Tim Haesebrouck

Ghent University

25 PUBLICATIONS 84 CITATIONS

SEE PROFILE

Some of the authors of this publication are also working on these related projects:

Project $\quad$ Algorithmic Sources of Publication Bias in QCA-Based Social-Scientific Research (Management, Political Science, Sociology) View project

Project The Politics of Multinational Military Operations View project 


\title{
An alternative update of the two-step QCA procedure
}

Tim Haesebrouck (Ghent University)

\begin{abstract}
In a recent contribution to Quality \& Quantity, Carsten Schneider (2018b) presents an updated version of the two-step QCA approach. Although Schneider (2018b) raises a lot of relevant issues, his revised procedure has three important disadvantages. First of all, the procedure sets the bar higher for remote conditions to be considered causally relevant than for proximate conditions. Second, the procedure makes it very difficult to find combinations of remote conditions. Third, the first step of the revised procedure does not build on a truth table, which makes it difficult to redesign the research if no meaningful results are found. This article proposes an alternative revision to the two-step approach, which aims to combine the best aspects of the original two-step procedure with the strengths of the revised procedure. In line with the original two-step approach, the first step of the procedure builds on a truth table. A new measure is added to the truth table of the first step, which provides an important parameter for coding the outcome column: cumulative coverage. The proposed procedure uses the same criteria for assessing the causal relevance of remote and proximate conditions, allows to straightforwardly uncover combinations of relevant remote conditions and helps researchers go back and forth between cases and evidence.
\end{abstract}




\section{Introduction}

In a recent contribution to Quality \& Quantity, Carsten Schneider (2018b) presents an updated version of the two-step QCA approach. The latter was introduced in an article by Schneider and Wagemann (2006) with two main goals: limiting the number of logical remainders and modelling the impact of contextual conditions. Although the idea of splitting up the QCAprocedure in two steps is an ingenious way to accomplish both objectives, the number of successful empirical applications of the method is relatively modest. Schneider (2018b) attributes this to the unclear set-theoretic status of the results of the first step of the procedure and suggests to redefine this step as an analysis of necessity.

Schneider (2018b) raises a lot of relevant issues in his analysis of the two-step procedure. One of the main problems with the original two step procedure is the lack of clear criteria for assessing the relevance of remote conditions. In line with the suggestion of Schneider (2018b), a revised two-step approach can draw on the criteria generally used to examine necessary conditions to solve this problem. However, three problems with the revised two-step procedure can keep researchers from finding the relevant remote conditions. First of all, the revised procedure sets the bar higher for remote conditions to be considered causally relevant than for proximate conditions. Second, the procedure makes it very difficult to find combinations of remote conditions. Third, the first step of the revised procedure does not build on a truth table, which makes it difficult to redesign the research if no meaningful results are found.

This article proposes an alternative revision to the two-step procedure, which aims to combine the best aspects of the original procedure with the strengths of the revisions suggested by Schneider (2018b). In line with the original two-step approach, the first step of the proposed 
procedure builds on a truth table. A new parameter for coding the outcome column is added to this truth table: cumulative coverage. This revision guarantees that the first step of the procedure results in a solution that is an (imperfect) superset of the outcome. The new procedure uses the same criteria for remote and proximate conditions to be considered causally relevant, allows to straightforwardly uncover combinations of remote conditions and go back and forth between cases and evidence.

The article is structured as follows. In line with the argument of Rohlfing and Zuber (2019) "that social scientists should be aware of philosophical theories of causation", the first section introduces regularity theories of causation as a possible theoretical underpinning of two step QCA. The second section demonstrates that the procedure suggested in Schneider (2018b) makes it difficult to discover contexts that are combinations of conditions and sets the bar very high for remote conditions to be considered causally relevant. The third section introduces an alternative procedure, which adds the cumulative coverage sufficiency (or consistency necessity) parameter to the truth table. Lastly, the conclusion recapitulates the main arguments of the contribution.

\section{Two step QCA and regularity theory}

In order to make an argument on whether or not two-step QCA is capable of identifying relevant remote conditions, a clear definition of relevance is needed. This article focusses on causal relevance. Although QCA can be used for other purposes, researchers should definitely not refrain from using causal language when applying QCA. In fact, it has been argued that one of the distinctive features of the method is that "it aims at causal interpretation" (Schneider and Wagemann 2012,8). Methods that want to draw conclusions on causal relations must be 
underpinned by a clear theory of causation, which offers a clear definition of causality and criteria for identifying causally relevant conditions.

Although several methodologists are skeptical on the practical implications of undergirding QCA with regularity theories (cf. Schneider 2018a), the latter constitutes the most obvious theory for underpinning QCA. First of all, a lot of publications on QCA refer to this theory or use concepts derived from this theory (e.g. Schneider and Wagemann 2012; Ragin 2009; Rohlfing and Zuber 2019). Moreover, the work of Baumgartner (2015) shows that it is possible to apply QCA in a consistent way with regularity theory. Finally, an alternative, fully developed, theory of causation that could underpin QCA has not yet been spelled out (Schneider 2018a; Rohlfing and Zuber 2019).

Regularity theories satisfy a number of principles that are in line with pre-theoretic intuitions regarding causal relations (Baumgartner 2008, 328). First of all, "regularity theories assume that the same cause is always accompanied by the same effect" (Graßhoff and May 2001, 85). This deterministic view of causation suggests that causally relevant conditions are part of sufficient combinations of conditions. Because of the complexity of reality, we can only know "certain elliptical or gappy universal laws" (Mackie 1974, 66). In consequence, we can only hope to find parts of a cause, not the full cause. Mackie's $(1965,247)$ definition of a causally relevant condition as an "Insufficient but Necessary part of an Unnecessary but Sufficient condition" (or an INUS-condition) makes clear that only indispensable parts of sufficient combinations can be considered causally relevant. This is because sufficient combinations can be conjunctively supplemented by arbitrary factors without altering their status as sufficient combination (Baumgartner 2015, 842). 
Second, regularity theories assume that an effect only occurs if a cause is present (Graßhoff and May 2001, 88). This implies that the disjunction of all sufficient combinations is necessary for an outcome. In other words, causally relevant factors are indispensable parts of sufficient conjuncts of a necessary disjunction. To avoid causal fallacies, only indispensable parts of sufficient conjuncts that cover at least one case (and are thus indispensable) can be considered causally relevant (Graßhoff and May 2001, 88; Baumgartner 2008). As illustrated by the Manchester-Factory-Hooters example of Mackie (1974, 83), sufficient combinations that are not indispensable parts of necessary disjunctions can be the result of spurious regularities. The latter do not reflect a direct causal relationship, but are the consequence of a common cause between two factors.

To sum up, modern regularity theories define causally relevant factors as indispensable parts of sufficient combinations that are indispensable parts of a necessary disjunction. Conditions that meet this criterion make a difference for whether or not the effect occurs in at least one combination of conditions (Baumgartner and Ambühl Forthcoming, 3-4). Regularity theories of causation, thus, consider causes as difference-makers for their effects.

The work of Michael Baumgartner (2006, 2009, 2015) shows that regularity theories of causation can undergird QCA. Two-step QCA does not constitute an exception in this regard. "The backbone of the two-step approach is the distinction between remote and proximate conditions" (Schneider 2018b). Remote conditions are relatively stable over time, temporarily and spatially remote from the outcome and outside the influence of present actors (Schneider and Wagemann 2006, 760). In their original formulation of the two-step approach, Schneider and Wagemann (2006, 761) argue that these conditions should be considered "outcome- 
enabling". Different combinations of these conditions can constitute contexts that make the outcome possible. In his reformulation of the two-step procedure, Schneider (2018b) argues that these contexts should be considered necessary conditions. More specifically, he argues that a disjunction of different (combinations of) conditions can be necessary contexts for an outcome. Conditions that are part of such a necessary disjunction are referred to as SUIN conditions: a sufficient but unnecessary part of an insufficient but necessary disjunction. After the first step assesses which remote conditions are SUIN-conditions, the second step consists of an analysis of sufficiency that includes the identified SUIN-conditions and the proximate conditions. The latter are temporarily and spatially closer to the outcome, vary over time and are subject to changes introduced by actors (Schneider and Wagemann 2006, 760). The result of this step reveals which proximate factors line up with the SUIN-conditions to form sufficient combinations for the outcome.

From the point of view of regularity theory, necessary conditions and SUIN-conditions cannot be causally interpreted (Thiem 2016). Moreover, the revised procedure sets the bar higher for remote conditions than for proximate conditions to be considered relevant (cf. infra). In consequence, it is not entirely clear whether the first step of the two-step procedure identifies causally relevant conditions or pursues another objective. The first step of the procedure presented in this article has a very clear objective: identifying causally relevant remote conditions. This corresponds to what Goertz (1994) refers to as "context as cause", which is one of three possible modes of context, next to "context as barrier" and "context as changing meaning" (Goertz 1994, 3-4; 14-51). The definition of "context as cause" closely resembles the definition of causal relevance derived from regularity theory: "the context is neither individually 
necessary nor sufficient, but in conjunction with other factors it explains the outcome or makes it more likely". In this category, contexts have "the same causal interpretation" as proximate conditions (Goertz 1994, 3). This is in line with regularity theories of causation, in which causal relevance is binary: a factor is an indispensable parts of sufficient combinations that are indispensable parts of a necessary disjunction or it is not. The definition of causation derived from regularity theory can be applied to both remote and proximate conditions. An assumption of two-step QCA is that there exist contextual factors that are relevant for explaining an outcome (Schneider 2018b). If this is the case, there should at least be one indispensable remote condition in each indispensable sufficient combination. Likewise, given that two-step QCA assumes that remote conditions are not sufficient on their own, at least one proximate conditions should be an indispensable parts of each indispensable sufficient combination.

Regularity theories of causation, thus, suggest that remote and proximate conditions have to meet the same criteria for being considered causally relevant. Nevertheless, there are two good reasons to split the QCA-analysis in two separate steps. First of all, model ambiguity tends to increase as more conditions are included in a QCA (Baumgartner and Thiem 2017). Splitting up the analysis reduces the numbers of conditions included each step, reducing the risk that the results become so ambiguous that they cannot be causally interpreted. More specifically, in line with the assumption that the outcome is best explained by a combination of remote and proximate conditions (Schneider 2018b), the two-step procedure excludes models that do not include remote conditions.

Second, in line with the original goal of the two-step procedure, splitting up the analysis reduces the number of logical remainders. Given that regularity theories suggest that only the 
parsimonious solution provides evidence for causal relevance and there are procedures for producing this formula that do not require assumptions on logical remainders, it is often assumed that limited diversity constitutes less of a problem from the perspective of regularity theory. However, in a recent paper, Haesebrouck (2019) demonstrates that the number of conditions for which the analysis neither shows that they are relevant or contextually irrelevant tends to increase as there are more logical remainders. In consequence, limited diversity reduces the explanatory power of a QCA.

\section{Revised two-step QCA}

This section examines whether the revised two-step procedure is capable of identifying causally relevant conditions. The second step of this procedure corresponds to an analysis of sufficiency, which is similar to the standard QCA procedure. Therefore, the focus is on the first step of the analysis. To test whether this step is capable of identifying causally relevant conditions, this section applies a variation on the inverse search strategy (Baumgartner 2015). This procedure starts with assuming a causal structure, producing data in line with this structure and testing whether an analytical procedure is capable of finding the assumed causal structure. Rather than working with artificial data, this article uses the original data of Haesebrouck (2015a). This data, with some small changes, was also used by Schneider (2018b) to illustrate the revised procedure. The first part of this section examines the causal relevance of the remote conditions in the analysis of Haesebrouck (2015a), the second part assesses whether the revised two-step procedure is capable of finding the relevant remote conditions, after which the third section discusses the general problems with the revised two step procedure. 


\subsection{Causal relevance and remote conditions}

The first step of Haesebrouck's (2015a) analysis identified two combinations of remote conditions that are relevant for the outcome large personnel contribution (LC), which each consisted of two conditions: (1) military stretch (MS) and military capacity (MC) and (2) military stretch (MS) and prior peacekeeping involvement (PI). The parsimonious solution of the second step of Haesebrouck's (2015a) analysis shows that conjunct (1) is sufficient in combination with a left-leaning executive (LE); conjunct (2) in combination with a left-leaning parliament (LP), resulting in the following formula: $\sim \mathrm{MS}^{*} \mathrm{MC}{ }^{*} \mathrm{LE}(1)+\sim \mathrm{MS}^{*} \mathrm{PI}{ }^{*} \mathrm{LP}(2) \rightarrow$ $\mathrm{MC}$

Two parameters of fit can be used to assess the causal relevance of conditions: consistency and coverage. Consistency indicates the degree to which the empirical data confirms that a condition is perfectly sufficient or necessary, coverage the extent to which it accounts for the behavior of the outcome (Haesebrouck 2015b). Both parameters are derived from fuzzy set theory. A sufficient condition constitutes a subset of the outcome; a necessary condition a superset. Consistency sufficiency indicates the extent to which a condition is a subset of the outcome, consistency necessity the extent to which it is a superset. Conversely, coverage sufficiency indicates the extent to which a condition is a superset of the outcome, coverage necessity the extent to which it is a subset. The coverage of a sufficient condition corresponds to its consistency as a necessary condition and vice versa.

Consistency and coverage can be used to examine whether or not a condition is an indispensable part of a sufficient combination that is an indispensable part of a necessary disjunction. A condition can be assumed to be causally relevant if removing it from a combination results in a 
substantial drop in the combination's consistency sufficiency. A condition is most clearly relevant if consistency falls below 0.75 , which is generally considered the lowest bound consistency value for a sufficient combination. If removing a sufficient combination from a necessary disjunction results in a drop in the coverage sufficiency of a solution, this indicates that the combination is an indispensable part of the disjunction. There is no generally accepted lower bound value for coverage, but there seems to be no reason to take another value than 0.75 .

Next to looking at consistency values, the cases covered by the solutions are also important for assessing whether conditions are causally relevant. Cases with a score above 0.5 in a solution and below 0.5 in the outcome strongly contradict sufficiency. Conversely, cases with a score below 0.5 in a condition and above 0.5 in the outcome contradict the necessity of the solution. If the number of contradictory cases covered by a sufficient combination increases if a condition is dropped, this suggests that the latter is causally relevant. If the number of contradictory cases necessity increases if a sufficient combination is dropped, the combination is indispensable.

First, regularity theory requires assessing whether each sufficient combination is an indispensable part of a necessary disjunction. ${ }^{1}$ The solution has a coverage sufficiency of 0.75 and there is one case it does not cover. If sufficient combination (2) is dropped from the disjunction, the coverage drops to 0.65 and one additional case with a score above 0.5 in the outcome is not covered by the solution. If sufficient combination (1) is dropped from the disjunction, the coverage drops to 0.37 and the solution does not cover 5 cases of the outcome. This confirms that both sufficient combinations are essential parts of the necessary disjunction.

\footnotetext{
${ }^{1}$ The analyses in this article use R packages QCA 3.3 (Duşa 2018) and SetMethods 2.3.1 (Oana et al. 2018).
} 
Table 1 Relevance of disjunctions

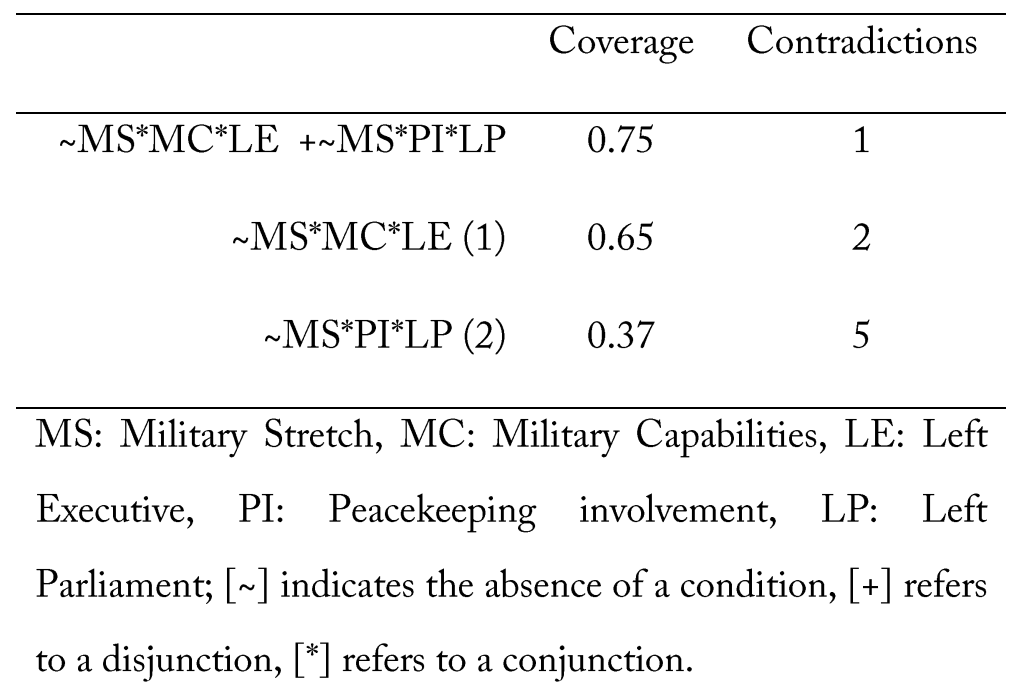

Subsequently, the relevance of the remote conditions in each sufficient combination is assessed. Table 2 shows that dropping one or both remote conditions from the sufficient combinations results in a drop in consistency and an increase in the number of contradictions. In most cases, consistency drops below 0.75 if a remote condition is removed, providing strong support that they are indispensable parts of their sufficient combination. Removing $\sim \mathrm{MS}$ from sufficient combination (1) only causes a drop of 0.03 in the combination's consistency, which at 0.85 is still far above 0.75 . Given that QCA is generally considered to be case-oriented and there is an additional contradictory case if $\sim \mathrm{MS}$ is dropped, there are good reasons to consider it an indispensable part of this combination.

To sum up, the remote conditions that are included in the solution of Haesebrouck (2015a) are indispensable parts of sufficient combinations that are indispensable parts of necessary disjunctions and thus causally relevant. 
Table 2 Relevance of remote conditions

\begin{tabular}{|c|c|c|c|c|c|}
\hline & Consistency & Contradictions & & Consistency & Contradictions \\
\hline$\sim \mathrm{MS}^{*} \mathrm{MC}^{*} \mathrm{LE}$ & 0.88 & 0 & MS*PI*LP & 0.79 & 0 \\
\hline$\sim \mathrm{MS} * \mathrm{LE}$ & 0.69 & 3 & $\sim \mathrm{MS}^{*} \mathrm{LP}$ & 0.64 & 3 \\
\hline $\mathrm{MC}^{*} \mathrm{LE}$ & 0.85 & 1 & PI*LP & 0.51 & 4 \\
\hline LE & 0.53 & 6 & LP & 0.49 & 7 \\
\hline \multicolumn{6}{|c|}{ MS: Military Stretch, MC: Military Capabilities, LE: Left Executive, PI: Peacekeeping involvement } \\
\hline
\end{tabular}

\subsection{Causal relevance and the two-step procedure}

This section assesses whether the revised two-step procedure is capable of finding the combinations of causally relevant remote conditions identified in the previous section. The first step of the revised procedure consists of an analysis of necessity. This involves testing whether single necessary conditions or disjunctions of necessary conditions meet a number of criteria. First of all, Schneider (2018b) suggests they must have a consistency of at least 0.9. Second, two parameters are used to assess whether these conditions are trivial: coverage and RoN. There are no fixed lower bound thresholds for these measures. Schneider (2018b) does suggest that "research practice indicates that RoN values lower than 0.5 are reason for concern". Schneider and Wagemann $(2012,146)$, in turn, argue that coverage values below 0.5 are rarely seen for necessary conditions. This suggests that 0.5 can be considered a lower bound value for both measures of relevance, although in most research practices a higher value would be preferred. In addition to these parameters, researchers are advised to examine whether there are deviant cases consistency necessity. Finally, when dealing with disjunctions, consistent necessary 
conditions should only be considered necessary contexts if "a theoretically meaningful concept can be formulated of which the disjuncts are functional equivalents" (Schneider 2018b).

When analyzing the original data of Haesebrouck (2015a) with the same thresholds as Schneider (2018b) (i.e. consistency 0.9, coverage 0.6 and RoN 0.5), the procedure does not find any necessary conditions. Only if the threshold for $\mathrm{RoN}$ is dropped below 0.5 and the threshold for coverage below 0.6 , a necessary disjunction would be found: $\mathrm{MC}+\mathrm{PI} \leftarrow \mathrm{LC}$. Given that both RoN and coverage are low, many researchers would conclude that the data does not confirm the relevance of these factors (or would not find this disjunction because they did not drop the RoN-threshold below 0.5 ).

Table 3 Analysis necessity remote conditions

\begin{tabular}{rccc}
\hline & Consistency & Coverage & RoN \\
\hline MC+PI & 0.91 & 0.52 & 0.47 \\
$\sim \mathrm{MS}$ & 0.87 & 0.57 & 0.61 \\
$\sim \mathrm{MS}{ }^{*} \mathrm{MC}$ & 0.69 & 0.74 & 0.87 \\
$\sim \mathrm{MS}{ }^{*} \mathrm{PI}$ & 0.39 & 0.78 & 0.95 \\
$\sim \mathrm{MS}^{*} \mathrm{MC}+\sim \mathrm{MS}{ }^{*} \mathrm{PI}$ & 0.79 & 0.72 & 0.82
\end{tabular}

MS: Military Stretch, MC: Military Capabilities, PI: Peacekeeping Involvement, [ ] indicates the absence of a condition, $[+]$ refers to a disjunction, $\left[{ }^{*}\right]$ refers to a conjunction.

The other causally relevant condition, MS, would only be discovered if the consistency value would be dropped below 0.9 . The coverage and RoN of this condition are relatively low and 
there is one contradictory case. In consequence, few researchers would consider this a necessary condition.

Finally, to find the combination of $\sim \mathrm{MS}$ and MC, the consistency value would have to be dropped to 0.69 ; to find the second combination, consistency would have to dropped to 0.39 . Obviously, few researchers would consider these combinations necessary conditions. The QCA 3.3 software is not capable of finding necessary disjunctions of combinations of conditions. However, even if it could, finding the two combinations of remote conditions would require a consistency threshold of 0.78 , which is generally considered far too low for a necessary condition.

\subsection{General problems two-step procedure}

Three general problems can explain why the two-step procedure is not capable of finding relevant (combinations of) remote conditions. First of all, the revised procedure sets the bar much higher for remote conditions than for proximate conditions. Second, it is very difficult to find contexts that are combinations of conditions with the new procedure. Third, the absence of a truth table in the first step of the revised procedure makes it difficult to redesign the research if no meaningful results are found.

First of all, one of the few weaknesses in Schneider's (2018b) article is that it does not provide a convincing theoretical or ontological argument for why remote conditions should be necessary conditions or part of necessary disjunctions, while proximate conditions should only be part of sufficient combinations. ${ }^{2}$ Schneider (2018b) argues that considering remote conditions as

\footnotetext{
${ }^{2}$ Rather than theoretical or ontological reasons, the most important motivation for remote conditions to be considered necessary is that they can disappear in the second step if they are not necessary or a part of a necessary
} 
necessary conditions was already suggested by the first version of two-step QCA, in which these were defined as "outcome-enabling" or as constituting "the context within which the outcome can occur" (Schneider and Wagemann 2006). However, given that "the logic of two-step QCA is such that it assumes the existence of contextual factors" (Schneider 2018b), the proximate conditions also only constitute combinations of conditions in which the outcome can occur, not combinations that fully explain the occurrence of the outcome. Neither remote or proximate conditions can be sufficient combinations on their own, but are part of sufficient conditions that combine remote and proximate factors. In other words, both can be considered indispensable parts of sufficient combinations that are indispensable parts of a necessary disjunction. Therefore, the same criteria should apply for assessing the causal relevance of remote and proximate conditions.

It is difficult to argue that the original data of Haesebrouck (2015a) does not support the relevance of the remote conditions without arguing that the data used by Schneider (2018b) does not support the relevance of some of the proximate conditions that are presented in his article. ${ }^{3}$ The parsimonious solution of the data used In Schneider (2018b) suggests that the combination of Military Capability with a Left Parliament and the combination of Peacekeeping Involvement with a Left Executive and Electoral Distance (ED) are sufficient for the outcome. If the same criteria for causal relevance would apply to the proximate conditions

\footnotetext{
disjunction. The procedure suggested in this article can also help researchers avoid that remote conditions disappear from the solution.

${ }^{3}$ Schneider (2018b) presents the intermediate solution. However, this solution would never be found if proximate conditions would have to meet the same criteria as remote conditions, given that it would require finding the necessary disjunction "LP+ PV+PV", which suggests either the presence of a left parliament or the presence or absence of parliamentary veto $(\mathrm{PV})$ is needed to produce the outcome.
} 
as to the remote conditions, $\mathrm{LP}+\mathrm{LE}^{*} \mathrm{ED}$ should be a necessary disjunction for the outcome. However, when using 0.9 as the consistency threshold and 0.5 as RoN and coverage threshold, only the second combination would be considered necessary for the outcome.

A second problem with the first step of the revised procedure is that it makes it very complicated to find conjuncts of necessary conditions. Although Schneider (2018b) is correct in arguing that "nothing prevents a SUIN condition to consist of two or more single remote factors combined by logical AND”, researchers face considerable obstacles when trying to find combinations of relevant remote conditions. Most importantly, the consistency necessity scores of combinations of conditions is generally lower than the scores of individual conditions. The solution's consistency necessity score is 0.79 , which would generally be considered too low for a necessary condition.

Schneider (2018b) explicitly argues that each "single conjunct must be a superset of the outcome", suggesting that the same thresholds apply to combinations of conditions as to individual conditions. To the best of my knowledge, this is the first time it has been argued that individual conditions cannot be considered necessary because their conjunction is not necessary. In consequence, it could be argued that it suffices for a combination of conditions to be considered necessary that its parts pass the consistency threshold. Researchers could first examine whether individual conditions or their disjunctions are necessary. Subsequently, these necessary conditions could be combined in combinations of conditions. With a consistency threshold of 0.87 , a RoN threshold of 0.4 and a coverage threshold of 0.5 , both $\mathrm{MC}+\mathrm{PI}$ and MS would be found as necessary condition, from which follows that " $\mathrm{MS}$ (MC+PI)" is necessary for the outcome. 
However, it would be a very cumbersome process to find combinations of necessary conditions in this way. First of all, one would need the exact right combination of consistency, coverage and RoN thresholds. A researcher that finds $\sim \mathrm{MS}$ as necessary condition after lowering the consistency threshold to 0.87 would probably not be inclined to also lower the RoN threshold to find an additional necessary disjunction. Likewise, after finding $\mathrm{MC}+\mathrm{PI}$ as a necessary disjunction, few researchers would drop the consistency threshold to find an additional necessary condition. It is particularly difficult to find combinations of remote conditions because the thresholds for consistency, coverage and RoN thresholds should not be fixed in a mechanical way. In research practice, these thresholds are generally inductively established, after looking at the results and assessing whether there are contradictory cases.

A third problem with the revised two-step procedure is that it does not use a truth table in the first step. One of the central features of QCA as a research approach is that it allows researchers to redesign earlier steps of their research project based on preliminary results. A crucial stage in this iterative process is the resolution of contradictory configurations. However, without a truth table, it is difficult to find the deviant cases that keep combinations of conditions or disjunctions of sufficient combinations from passing consistency and coverage thresholds or to find the most suitable consistency threshold. Finally, the truth table is an invaluable instrument for analyzing combinations of conditions.

\section{Towards a new two-step approach}

This section proposes an alternative two-step approach, which combines the strengths of the original and the revised procedure. The proposed procedure alleviates the three problems discussed above by using a truth table in the first step of the analysis. The main problem of the 
original procedure, the lack of clear criteria for coding a truth table row, is solved by introducing an indicator in the truth table that measures the extent to which a solution constitutes a superset of the outcome: cumulative coverage.

\subsection{Remote conditions as imperfect supersets}

In line with the revisions suggested by Schneider (2018b), the goal of the first step of the proposed procedure is identifying a solution that constitutes an (imperfect) superset of the outcome. If the assumption behind two-step QCA holds, there should be at least one remote condition that is indispensable in each indispensable sufficient combination. In consequence, at least one remote condition should be associated with each cases of the outcome and a disjunction of (combinations of) remote conditions should be a superset of the outcome (Schneider 2018b).

The proposed procedure allows for lower and less rigid consistency necessity thresholds than suggested in Schneider (2018b). There are three main reasons for allowing a lower threshold. First of all, one of the problems with the revised two-step procedure is that it uses other criteria for assessing the relevance of proximate conditions than for remote conditions. Being part of a necessary disjunction with a consistency above 0.9 is not a requirement for proximate conditions, so why would it be for remote conditions (cf. supra). A second reason for allowing thresholds below 0.9 is that combinations of conditions generally have lower consistency values than single conditions (cf. supra). Third, the main consequence of allowing for a lower consistency necessity in the first step is that the result of the second step will have a lower coverage sufficiency. The latter is generally not considered a problem in QCA. Schneider and Wagemann (2012, 139), for example, argue that there is no lower bound for coverage. 
Baumgartner and Ambühl (Forthcoming) do argue that a lower bound coverage value is required to allow for a causal interpretation of solutions. However, a coverage sufficiency threshold of 0.9 has never been suggested. In line with the consistency threshold for sufficient conditions, 0.75 seems to constitute an appropriate lower bound value for the coverage of a solution. A low coverage is more problematic if it is caused by deviant cases coverage, which are cases that are not covered by the solution and, thus, not explained by this solution (Schneider and Wagemann 2012, 280). However, if these deviant cases are limited in numbers and explicitly discussed, they do not completely invalidate the results of the research.

To sum up, a consistency necessity threshold below 0.9 does not seem problematic in the first step of the analysis, as long as the coverage value of the solution of the second step does not fall below 0.75 and there is only a (very) limited number of deviant cases coverage.

\subsection{The revised first step: introducing cumulative coverage}

In line with the original two-step procedure, this article suggests to use a truth table in the first step to identify causally relevant conditions. QCA is capable of finding combinations of causally relevant conditions by deriving the parsimonious solution from the truth table. However, there are two potential problems with applying this procedure to the first step.

First of all, in order to draw conclusions on causal relevance, causal homogeneity must be assumed: all causally relevant conditions that are not included in the analysis must be constant across all cases. This assumption is not satisfied in the first step of two-step QCA, because the proximate conditions are not included in the first step of the analysis. This could result in causal fallacies. More specifically, causal relevance can be attributed to an irrelevant remote condition because a causally relevant proximate condition was not included in the analysis. However, if 
one assumes that every sufficient cause includes at least one remote condition, at least one condition in each combination of remote conditions should be causally relevant even if proximate factors vary across the cases.

A second potential problem is that truth tables are generally used to identify sufficient combinations, but remote conditions are not assumed to be sufficient for the outcome. In consequence, the coding of the outcome column cannot solely be based on the consistency sufficiency of the truth table rows. However, this parameter does provide useful information on which rows include causally relevant remote conditions. The assumption that at least one remote condition is an indispensable part of each sufficient combination implies that rows in which the relevant remote conditions are missing will never be linked to the outcome and, thus, have a very low consistency level. These rows should be assigned a score of 0 in the outcome column, given that they do not include conditions that are parts of sufficient combinations.

Rows for which there is no evidence that they are insufficient cannot automatically be assumed to include relevant remote conditions. Therefore, the consistency sufficiency of a truth table row cannot be the only criterion for coding truth table rows. As argued above, and in line Schneider's (2018b) revised procedure, the extent to which a solution constitutes a superset of the outcome provides an important parameter for this. Only if the solution is a superset of the outcome, a remote condition will be part of every sufficient combination. Therefore, consistency necessity or coverage sufficiency provides an appropriate measure for coding the outcome column. Unlike consistency sufficiency, it is not relevant to assess the coverage sufficiency of single truth table rows. First of all, the coverage of combinations of conditions will generally be very low, given that cases are distributed amongst different truth table rows. Second, coverage 
and consistency tend to be inversely correlated, with high consistency coming at the price of low coverage and vice versa. Combinations of conditions that already come close to being sufficient and, thus, include relevant remote conditions, can have a relatively low coverage value. Moreover, combinations of conditions in which cases have high membership scores tend to have a higher coverage than combinations in which cases have low membership scores, irrespective of whether they are generally associated with the outcome or the absence of the outcome. For these reasons, the coverage value of a truth table row does not constitute a good criterion for the coding of the outcome column.

Coverage sufficiency will generally increase as more rows are included in the minimization of a truth table. The latter either results in dropping conditions from a combination or adding a disjunct to a solution, both of which tend to have a positive impact on the coverage of the solution. A new measure can be introduced in the truth table to assess whether the coverage of the solution will be sufficiently high: cumulative coverage. This indicator measures how much of the outcome will be explained if a row is included in the minimization process. Cumulative coverage can be calculated with the formula below, in which $\mathrm{M}_{\mathrm{row1}, . . \mathrm{x}}$ corresponds to the result of minimizing row $\mathrm{X}$ with the rows above row $\mathrm{X}$ and $\mathrm{Y}$ corresponds to the outcome. ${ }^{4}$

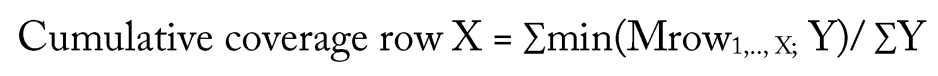

Consistency sufficiency and cumulative coverage provide parameters for coding the outcome column in the first step. This can be illustrated with the data of Haesebrouck (2015a). Table 4 represents the truth table of the remote conditions, with the cumulative coverage presented in

\footnotetext{
${ }^{4}$ Given that only the parsimonious solution can be causally interpreted according to regularity theories, logical remainders are included in the minimization process.
} 
the second to last column. ${ }^{5}$ In line with the coding of the outcome column in standard QCA practice, the choice of an appropriate level of consistency and coverage is specific to every research project and should be explicitly justified (Schneider and Wagemann 2012, 279).

Several criteria serve as guidelines for coding the outcome column. First of all, it seems advisable to keep both consistency and cumulative coverage above a minimum threshold. Deciding on a lower bound for consistency seems particularly tricky, given that the second step is expected to result in a rise in consistency. However, a consistency above 0.5 seems advisable, since this implies that there is more evidence in favor than against the claim of sufficiency. A low cumulative coverage indicates that a large portion of the outcome will not be covered by the combinations of remote conditions produced by the first step. This increases the probability that the result of the second step will include paths that do not include remote conditions. Moreover, the coverage of the result of the second step will almost always be lower (and never be higher) than the cumulative coverage threshold. In consequence, cumulative coverage levels above 0.8 are advisable.

When deciding on a consistency - cumulative coverage threshold, researchers should also examine the cases in the truth table rows. More specifically, researchers should avoid assigning a score of 0 to rows that include cases with a score above 0.5 in the outcome. These cases will not be covered by combinations of remote conditions that result from the first step, which shows

\footnotetext{
${ }^{5}$ Cumulative coverage cannot be produced with the QCA 3.3 software. However, it can straightforwardly be found by looking at the coverage of the solutions that are produced when a row is included in the minimization process (cf. replication material to this article).
} 
that the outcome can occur in the absence of these combinations. This, in turn, increases the probability that the solution will include paths without remote conditions.

Table 4 Truth table remote conditions

\begin{tabular}{ccccccccl}
\hline & & & & & & & Cumulative \\
Row & MC & PI & MS & GP & Outcome & Consistency & Coverage & Cases \\
\hline 1 & 1 & 0 & 0 & 1 & 1 & 0.913 & 0.23 & GR, IT \\
2 & 1 & 1 & 0 & 0 & 1 & 0.911 & 0.49 & FR, PT \\
3 & 1 & 1 & 0 & 1 & 1 & 0.832 & 0.52 & PL \\
4 & 0 & 1 & 0 & 0 & 1 & 0.678 & 0.59 & CZ, FI \\
5 & 1 & 0 & 0 & 0 & 1 & 0.612 & 0.79 & BE, DE, ES, NL \\
6 & 1 & 0 & 1 & 0 & 0 & 0.490 & 0.80 & GB \\
7 & 0 & 1 & 1 & 0 & 0 & 0.391 & 0.89 & AT, IE, SE \\
8 & 0 & 0 & 0 & 0 & 0 & 0.316 & 0.97 & LT, LU, LV, SI \\
9 & 0 & 1 & 1 & 1 & 0 & 0.265 & 0.99 & HU, SK \\
10 & 0 & 0 & 1 & 0 & 0 & 0.233 & 0.98 & EE \\
\hline
\end{tabular}

MS: Military Stretch, MC: Military Capabilities, GP: Geographic Proximity PI: Peacekeeping involvement

According to these guidelines, the cut-off point consistency-cumulative coverage can be placed between rows 5 and 6 . Row 5 meets the lower bound value for consistency and falls just below the lower bound for cumulative coverage. One problem with this threshold is that there is one deviant case: Ireland has a score above 0.5 in the outcome but is located in row 7 . The latter is 
an example of the two non-mutually exclusive types of truth table rows that correspond to contradictory configurations in the first step of two-step QCA. First of all, truth table rows with a very low consistency that include cases with a score above 0.5 in the outcome. Second, truth table rows with a low consistency that have a substantially higher cumulative coverage than the row above. Such contradictory rows indicate that there might be a remote condition missing, the population of interest must be respecified or that the measurement of the outcome or the conditions should be reconsidered (Schneider and Wagemann 2012, 120-123).

The truth table provides information on what strategies can be used to resolve contradictory configurations and arrive at a better understanding of the impact of remote conditions. If these strategies do not work, researchers can continue their analysis in two ways. First of all, the consistency cumulative coverage threshold could be dropped below this row, but this would result in two rows with a very low consistency to be included in minimization. Second, researchers can continue the analysis with the higher threshold, but explicitly mention the uncovered case when discussing their solutions. This was done in Haesebrouck (2015a), resulting in the solution discussed above. However, researchers should only continue their analysis without solving contradictory configurations if both the cumulative coverage and consistency are sufficiently high. If the truth table does not display acceptable consistency and cumulative coverage values, researchers should reconsider prior steps of their research project, including the decision to apply two-step QCA.

\subsection{The second step of the revised procedure}

Schneider (2018b) suggests a small revision to the second step of the procedure: including all remote conditions in one analysis. The major benefit of this revision is that the unique coverage 
values of sufficient combinations are not biased towards high values. An important downside is that the number of conditions will rise, which might cause researchers to miss relevant combinations of conditions and produce suboptimal solutions. This is because consistency levels are not monotomic in fuzzy set QCA: $A^{*} B$ can have a higher consistency value than $A^{*} B^{*} C$ (Baumgartner and Ambühl Forthcoming, 17).

The data of Haesebrouck (2015a) shows that this can result in situations in which researchers miss relevant combinations of conditions and produce suboptimal solutions. Table 5 presents the truth table that includes the three relevant remote conditions together with the proximate conditions. Table 6 presents the truth table with only the conditions from the first combination of remote conditions ( $\mathrm{MS}$ and $\mathrm{MC}$ ), table 7 the truth table with only the conditions from the second combination of remote conditions ( $\sim \mathrm{MS}$ and PI). The only difference between row 3 of table 6 and row 5 of table 5 is that the latter includes the absence of PI. The only difference between row 2 of table 7 and row 4 of table 5 is that the latter includes the absence of MC. However, row 3 of table 6 and row 2 of table 7 have a consistency that is sufficiently high for considering them sufficient for the outcome, whereas row 4 and 5 of table 5 do not.

Table 8 compares solution (1), which results from minimizing table 5, and solution (2), which results from disjunctively combining the results of minimizing table 6 and 7 . Solution (2) scores better on several criteria than solution (1). The consistency of solution (2) is lower than the consistency of solution (1), but still above the minimum 0.75 threshold, and its coverage is substantially higher. Moreover, solution (1) has three deviant cases coverage, compared to one in solution (2). In consequence, it is difficult to argue that solution (2) should not be preferred over solution (1) 
Table 5 Truth table second step all remote conditions

\begin{tabular}{|c|c|c|c|c|c|c|c|c|c|c|}
\hline Row & MS & $\mathrm{MC}$ & $\mathrm{PI}$ & LE & LP & PV & ED & Outcome & Consistency & cases \\
\hline 1 & 0 & 1 & 1 & 1 & 1 & 0 & 1 & 1 & 0.93 & FR,PL,PT \\
\hline 2 & 0 & 1 & 0 & 1 & 1 & 1 & 1 & 1 & 0.89 & $\mathrm{ES}$ \\
\hline 3 & 0 & 1 & 0 & 1 & 1 & 0 & 1 & 1 & 0.85 & BE,GR \\
\hline 4 & 0 & 0 & 1 & 1 & 1 & 1 & 1 & 0 & 0.74 & FI \\
\hline 5 & 0 & 1 & 0 & 1 & 0 & 0 & 1 & 0 & 0.67 & $\mathrm{IT}$ \\
\hline 6 & 0 & 0 & 0 & 1 & 1 & 0 & 1 & 0 & 0.63 & SI \\
\hline 7 & 1 & 0 & 1 & 1 & 1 & 1 & 1 & 0 & 0.54 & IE,SK \\
\hline 8 & 0 & 0 & 1 & 1 & 0 & 1 & 1 & 0 & 0.54 & $\mathrm{CZ}$ \\
\hline 9 & 1 & 0 & 1 & 0 & 1 & 1 & 1 & 0 & 0.46 & $\mathrm{HU}$ \\
\hline 10 & 0 & 1 & 0 & 0 & 0 & 1 & 1 & 0 & 0.44 & $\mathrm{DE}$ \\
\hline 11 & 0 & 0 & 0 & 1 & 1 & 1 & 1 & 0 & 0.43 & LU \\
\hline 12 & 1 & 1 & 0 & 1 & 0 & 0 & 1 & 0 & 0.38 & GB \\
\hline 13 & 0 & 0 & 0 & 0 & 0 & 1 & 1 & 0 & 0.36 & $\mathrm{LT}$ \\
\hline 14 & 1 & 0 & 1 & 1 & 1 & 1 & 0 & 0 & 0.25 & $\mathrm{AT}, \mathrm{SE}$ \\
\hline 15 & 1 & 0 & 0 & 0 & 0 & 1 & 1 & 0 & 0.24 & $\mathrm{EE}$ \\
\hline 16 & 0 & 0 & 0 & 0 & 1 & 1 & 0 & 0 & 0.22 & LV \\
\hline 17 & 0 & 1 & 0 & 0 & 0 & 1 & 0 & 0 & 0.14 & NL \\
\hline
\end{tabular}

MS: Military Stretch, MC: Military Capabilities, PI: Peacekeeping Involvement, LE: Left Executive, LP: Left Parliament; PV: Parliamentary Veto; ED: Electoral Distance 
Table 6 Truth table second step MS*MC

\begin{tabular}{|c|c|c|c|c|c|c|c|c|c|}
\hline Row & MS & $\mathrm{MC}$ & $\mathrm{LE}$ & LP & PV & ED & Outcome & Consistency & Cases \\
\hline 1 & 0 & 1 & 1 & 1 & 1 & 1 & 1 & 0.923 & ES \\
\hline 2 & 0 & 1 & 1 & 1 & 0 & 1 & 1 & 0.865 & BE,FR,GR,PL,PT \\
\hline 3 & 0 & 1 & 1 & 0 & 0 & 1 & 1 & 0.748 & IT \\
\hline 4 & 0 & 0 & 1 & 1 & 0 & 1 & 0 & 0.735 & SI \\
\hline 5 & 0 & 0 & 1 & 0 & 1 & 1 & 0 & 0.592 & $\mathrm{CZ}$ \\
\hline 6 & 0 & 0 & 1 & 1 & 1 & 1 & 0 & 0.573 & FI,LU \\
\hline 7 & 1 & 1 & 1 & 0 & 0 & 1 & 0 & 0.547 & GB \\
\hline 8 & 0 & 1 & 0 & 0 & 1 & 1 & 0 & 0.543 & $\mathrm{DE}$ \\
\hline 9 & 1 & 0 & 1 & 1 & 1 & 1 & 0 & 0.509 & IE,SK \\
\hline 10 & 0 & 0 & 0 & 0 & 1 & 1 & 0 & 0.457 & $\mathrm{LT}$ \\
\hline 11 & 1 & 0 & 0 & 1 & 1 & 1 & 0 & 0.388 & $\mathrm{HU}$ \\
\hline 12 & 1 & 0 & 0 & 0 & 1 & 1 & 0 & 0.354 & $\mathrm{EE}$ \\
\hline 13 & 0 & 0 & 0 & 1 & 1 & 0 & 0 & 0.237 & LV \\
\hline 14 & 1 & 0 & 1 & 1 & 1 & 0 & 0 & 0.226 & AT,SE \\
\hline 15 & 0 & 1 & 0 & 0 & 1 & 0 & 0 & 0.167 & NL \\
\hline
\end{tabular}

MS: Military Stretch, MC: Military Capabilities, LE: Left Executive, LP: Left Parliament; PV: Parliamentary Veto; ED: Electoral Distance 
Table 7 Truth table second step MS*PI

\begin{tabular}{|c|c|c|c|c|c|c|c|c|c|}
\hline Row & MS & $\mathrm{PI}$ & $\mathrm{LE}$ & LP & PV & $\mathrm{ED}$ & Outcome & Consistency & cases \\
\hline 1 & 0 & 1 & 1 & 1 & 0 & 1 & 1 & 0.90 & FR,PL,PT \\
\hline 2 & 0 & 1 & 1 & 1 & 1 & 1 & 1 & 0.76 & FI \\
\hline 3 & 0 & 0 & 1 & 1 & 0 & 1 & 0 & 0.71 & BE,GR,SI \\
\hline 4 & 0 & 0 & 1 & 0 & 0 & 1 & 0 & 0.62 & IT \\
\hline 5 & 0 & 0 & 1 & 1 & 1 & 1 & 0 & 0.56 & ES,LU \\
\hline 6 & 1 & 1 & 1 & 1 & 1 & 1 & 0 & 0.54 & IE,SK \\
\hline 7 & 0 & 1 & 1 & 0 & 1 & 1 & 0 & 0.54 & $\mathrm{CZ}$ \\
\hline 8 & 1 & 1 & 0 & 1 & 1 & 1 & 0 & 0.46 & $\mathrm{HU}$ \\
\hline 9 & 1 & 0 & 1 & 0 & 0 & 1 & 0 & 0.32 & GB \\
\hline 10 & 0 & 0 & 0 & 0 & 1 & 1 & 0 & 0.31 & DE,LT \\
\hline 11 & 1 & 0 & 0 & 0 & 1 & 1 & 0 & 0.27 & $\mathrm{EE}$ \\
\hline 12 & 1 & 1 & 1 & 1 & 1 & 0 & 0 & 0.25 & AT,SE \\
\hline 13 & 0 & 0 & 0 & 1 & 1 & 0 & 0 & 0.22 & LV \\
\hline 14 & 0 & 0 & 0 & 0 & 1 & 0 & 0 & 0.12 & NL \\
\hline
\end{tabular}

MS: Military Stretch, PI: Peacekeeping Involvement, LE: Left Executive, LP: Left Parliament; PV:

Parliamentary Veto; ED: Electoral Distance 


\section{Deviant Cases}

Solution Consistency Coverage Consistency Coverage

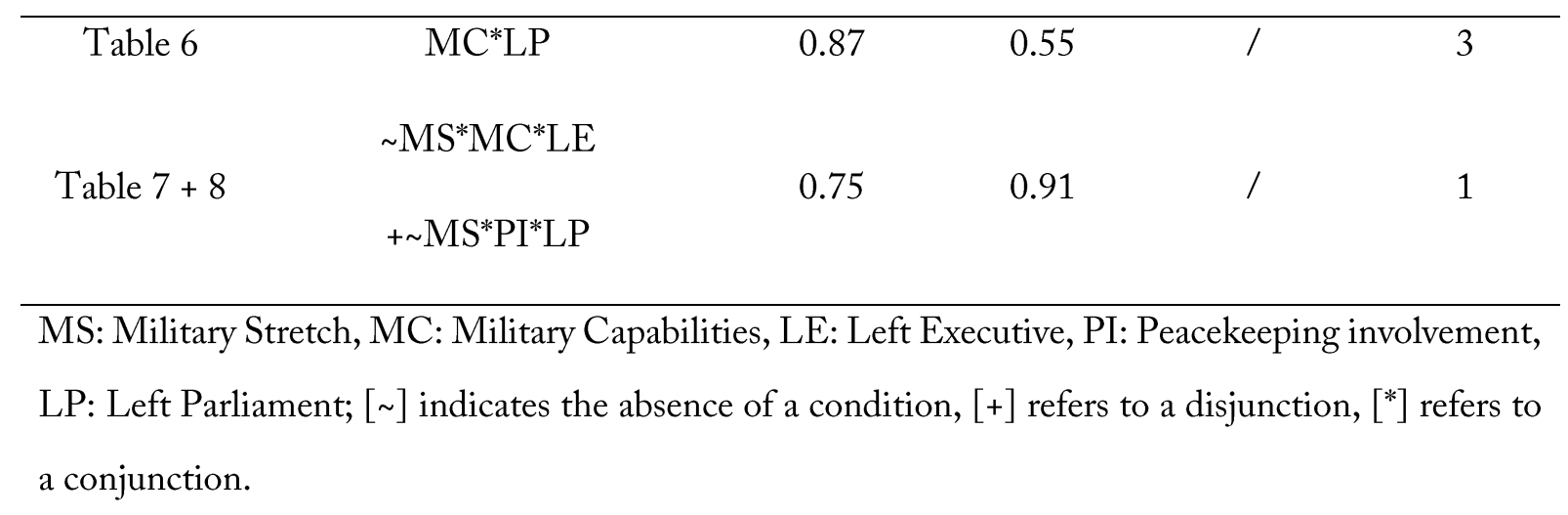

The problem identified by Schneider (2018b), the unduly high unique coverage of sufficient combinations, can be straightforwardly solved by taking into account all sufficient paths when calculating the unique coverage. More specifically, the unique coverage of a path can be calculated by subtracting the coverage of the disjunction of all other paths from the total coverage of the solution. This results in a unique coverage of $0.54(0.91-0.37)$ for $\sim \mathrm{MS}^{*} \mathrm{MC} \mathrm{C}^{*} \mathrm{LE}$ and a unique coverage of $0.22(0.91-0.69)$ for $\sim \mathrm{MS}^{*} \mathrm{PI}^{*} \mathrm{LP}{ }^{6}$

\section{Conclusions}

This article agrees with Schneider (2018b) that the two-step QCA procedure is a valuable and underused variant of QCA. His revised procedure has a lot of merit, most importantly it introduces a clear criterion for assessing the relevance of remote conditions in the first step of the analysis. This procedure might be the most appropriate choice in specific research situations,

\footnotetext{
${ }^{6}$ These values are substantially lower than the corresponding values when both solutions are studied In isolation, respectively 0.69 and 0.37 .
} 
for example for assessing whether theoretical hunches about specific (disjunctions of) remote conditions are correct. However, the procedure suggested in the present article has a number of benefits. Most importantly, by building on a truth table in the first step of the analysis, it allows to more straightforwardly find combinations of conditions and go back and forth between cases and evidence. By introducing the cumulative coverage measure, researchers get clear guidelines for coding the outcome column during the first step of the analysis. In conclusion, it is important to stress yet another point of agreement with Schneider (2018b): the choice of twostep QCA must rest on a strong theoretical argument that the outcome is best explained by the combination of remote and proximate conditions. Given that the two-step procedure does not provide conclusive evidence that this is the case, the results are dependent on whether or not this assumption holds. 


\section{References}

Baumgartner, M.: Complex Causal Structures. Extensions of a Regularity Theory of Causation,. University of Bern (2006)

Baumgartner, M.: Regularity theories reassessed. Philosophia 36(3), 327-354 (2008)

Baumgartner, M.: Inferring causal complexity. Sociological methods \& research 38(1), 71$101(2009)$

Baumgartner, M.: Parsimony and causality. Quality \& Quantity 49(2), 839-856 (2015)

Baumgartner, M., Ambühl, M.: Causal modeling with multi-value and fuzzy-set Coincidence Analysis. Political Science Research and Methods, 1-17 (Forthcoming). doi:10.1017/psrm.2018.45

Baumgartner, M., Thiem, A.: Model ambiguities in configurational comparative research. Sociological Methods \& Research 46(4), 954-987 (2017)

Duşa, A.: QCA with R: A Comprehensive Resource. Springer, Cham (2018)

Goertz, G.: Contexts of international politics. Cambridge University Press, Cambridge (1994)

Graßhoff, G., May, M.: Causal regularities. In: Spohn, W., Ledwig, M., Esfeld, M. (eds.) Current issues in causation. pp. 85-114. Mentis, Hardehausen (2001)

Haesebrouck, T.: Democratic Contributions to UN Peacekeeping Operations. A Two-Step Fuzzy Set QCA of Unifil II. Romanian Journal of Political Science 15(1) (2015a)

Haesebrouck, T.: Pitfalls in QCA's consistency measure. Journal of Comparative Politics(2), $65-80(2015 b)$ 
Haesebrouck, T.: Relevant, irrelevant or ambiguous? Towards a correct interpretation of QCA's solution types. Unpublished manuscript (2019)

Mackie, J.: Causes and conditions. American philosophical quarterly 2(4), 245-264 (1965)

Mackie, J.: The cement of the universe: A study of causation. Oxford University Press, (1974)

Oana, I.-E., Medzihorsky, J., Quaranta, M., Schneider, C.Q., Oana, M.I.-E.: Package 'SetMethods'. (2018)

Ragin, C.: Redesigning social inquiry: Fuzzy sets and beyond. University of Chicago Press, (2009)

Rohlfing, I., Zuber, C.I.: Check Your Truth Conditions!Clarifying the Relationship between Theories of Causation and Social Science Methods for Causal Inference. Sociological Methods \& Research (2019). doi:10.1177/0049124119826156

Schneider, C.Q.: Realists and Idealists in QCA. Political Analysis 26(2), 246-254 (2018a)

Schneider, C.Q.: Two-step QCA revisited: the necessity of context conditions. Quality \& Quantity, 1-18 (2018b)

Schneider, C.Q., Wagemann, C.: Reducing complexity in Qualitative Comparative Analysis (QCA): Remote and proximate factors and the consolidation of democracy. European Journal of Political Research 45(5), 751-786 (2006)

Schneider, C.Q., Wagemann, C.: Set-theoretic methods for the social sciences: A guide to qualitative comparative analysis. Cambridge University Press, (2012)

Thiem, A.: Standards of good practice and the methodology of necessary conditions in qualitative comparative analysis. Political analysis 24(4), 478-484 (2016) 